\title{
On wide Aronszajn trees in the presence of MA
}

\author{
Mirna Džamonja* Saharon Shelah ${ }^{\dagger}$
}

June 1,2020

\begin{abstract}
A wide Aronszajn tree is a tree of size and height $\omega_{1}$ with no uncountable branches. We prove that under $M A\left(\omega_{1}\right)$ there is no wide Aronszajn tree which is universal under weak embeddings. This solves an open question of Mekler and Väänänen from 1994.

We also prove that under $M A\left(\omega_{1}\right)$, every wide Aronszajn tree weakly embeds in an Aronszajn tree, which combined with a result of Todorčević from 2007, gives that under $M A\left(\omega_{1}\right)$ every wide Aronszajn tree embeds into a Lipschitz tree or a coherent tree. We also prove that under $M A\left(\omega_{1}\right)$ there is no wide Aronszajn tree which weakly embeds all Aronszajn trees, improving the result in the first paragraph as well as a result of Todorčević from 2007 who proved that under $M A\left(\omega_{1}\right)$ there are no universal Aronszajn trees. 11
\end{abstract}

\section{Introduction}

We study the class $\mathcal{T}$ of trees of height and size $\aleph_{1}$, but with no uncountable branch. We call such trees wide Aronszajn trees. A particular instance of such a tree is a classical Aronszajn tree, so the class $\mathcal{A}$ of Aronszajn trees satisfies $\mathcal{A} \subseteq \mathcal{T}$. Apart from their

\footnotetext{
*School of Mathematics, University of East Anglia, Norwich, NR4 7TJ, United Kingdom, M.Dzamonja@uea.ac.uk, https://www.logiqueconsult.eu

${ }^{\dagger}$ Department of Mathematics, Hebrew University of Jerusalem, 91904 Givat Ram, Israel, shelah@math.huji.ac.il, http://shelah.logic.at

${ }^{1}$ Mirna Džamonja thanks l'Institut d'Histoire et de Philosophie des Sciences et des Techniques, CNRS et Université PanthÂl'on-Sorbonne, Paris, where she is an Associated Member, and she thanks the Hebrew University of Jerusalem for their hospitality in April 2019. Saharon Shelah thanks the Israel Science Foundation for their grant 1838/19 and the European Research Council for their grant 338821. The authors thank Tanmay Inamdar for many very constructive comments and the anonymous referee for a prompt and careful reading and, moreover, providing them with the enclosed diagrams in Latex. They would also like to acknowledge that after reading this preprint, Stevo Todorčević informed them that upon closer inspection one can see that his arguments regarding Lipschitz trees in [1] reveal that the fact that the trees have countable levels is not used, and therefore one could use [11] combined with [12] to solve the Mekler-Väänänen problem.

This is publication number 1186 in Shelah's publication list.
} 
intrinsic interest in combinatorial set theory, these classes are also interesting from the topological point of view, since they give rise to a natural generalisations of metric spaces, $\omega_{1}$-metric spaces introduced by Sikorski in [9] and further studied in [7], [1] or [3], for example. The $\omega_{1}$-distance function in trees is given by the $\Delta$-function, which is defined by $\Delta(x, y)=\operatorname{ht}\left(x \cap_{T} y\right)$ for $x \neq y$ and $\Delta(x, x)=0$. Here $x \cap_{T} y$ represents the meet in the tree (as our trees will be trees of sequences of ordinals, this notation is more natural than $\wedge_{T}$ ). Classes $\mathcal{T}$ and $\mathcal{A}$ can be quasi-ordered using the notion of weak embedding, which is defined as follows:

Definition 1.1 For two trees $T_{1}, T_{2}$, we say that $T_{1}$ is weakly embeddable in $T_{2}$ and we write $T_{1} \leq T_{2}$, if there is $f: T_{1} \rightarrow T_{2}$ such for all $x, y \in T_{1}$

$$
x<_{T_{1}} y \Longrightarrow f(x)<_{T_{2}} f(y) .
$$

We are interested in the structure of $(\mathcal{T}, \leq)$ and $(\mathcal{A}, \leq)$. In particular, we address the question of the existence of a universal element in these classes. This is of special interest since among the many interesting and correct results of the paper [7] from 1993 there is also a claim that $M A\left(\omega_{1}\right)$ implies that there is a universal element in $(\mathcal{T}, \leq)$, the argument for which was soon after found to be faulty. Ever since, the status of the possible existence of a universal element in $(\mathcal{T}, \leq)$ under $M A\left(\omega_{1}\right)$ has remained an open question.

Our first result is Theorem 4.1, which proves that under $M A\left(\omega_{1}\right)$ there is no universal element in $(\mathcal{A}, \leq)$. This gives an alternative proof to a result of Todorčević from [12], whose Theorem 4.3.34 proves the same using the class of coherent trees. For more on this see $\$ 2$.

The second result is Theorem 5.1, which shows that under $M A\left(\omega_{1}\right)$ every wide Aronszajn tree weakly embeds into an Aronszajn tree. Putting the two results together, we obtain the main result of the paper, Theorem 6.1, which shows that under $M A\left(\omega_{1}\right)$ the class $(\mathcal{T}, \leq)$ has no universal element. This resolves the question raised by [7].

Combining our result with Lemma 4.3.32 from [12], we obtain that under $M A\left(\omega_{1}\right)$ every wide Aronszajn tree weakly embeds into a coherent tree, or equivalently under $M A\left(\omega_{1}\right)$, into a Lipschitz tree (Corollary 6.2(1)). We also obtain (Corollary 6.2 (2)) a strengthening of Todorčević's result about the non-existence of universal Aronszajn trees under $M A\left(\omega_{1}\right)$, namely we prove that under $M A\left(\omega_{1}\right)$ not even the class of wide Aronszajn trees suffices to weakly embed all Aronszajn (or all coherent) trees.

\section{Some facts about $(\mathcal{T}, \leq)$ and $(\mathcal{A}, \leq)$}

Note that if there is a weak embedding from a tree to another, then there is one which preserves levels (see Observation 3.4), so we may restrict our attention to such embeddings.

An important idea of Đuro Kurepa in [5] (see [6] for a complete edition) is that of a functor now known as $\sigma$-functor. This functor associates to a tree $T$ the tree $\sigma T$ of the 
increasing sequences of $T$, ordered by inclusion. The basic fact is that there cannot be a weak embedding from $\sigma T$ to $T$. If $T$ has no uncountable branch, neither does $\sigma T$, but even if the cardinality of $T$ is $\aleph_{1}$, the cardinality of $\sigma T$ is $2^{\aleph_{0}}$. However, when $C H$ holds, for any $T \in \mathcal{T}$ we have $\sigma T \in \mathcal{T}$ and similarly for $\mathcal{A}$. Therefore, under $C H$ neither class $(\mathcal{T}, \leq)$ nor $(\mathcal{A}, \leq)$ have a universal element.

Todorčević studied level-preserving functions $f$ between trees which satisfy the Lipschitz condition

$$
\Delta_{T_{1}}(x, y) \leq \Delta_{T_{2}}(f(x), f(y)) .
$$

We may think of Lipschitz embeddings as contractions. This notion led Todorčević to introduce a subclass of $\mathcal{A}$ which consists of those Aronszajn trees on which every levelpreserving map from an uncountable subset of $T$ into $T$, has an uncountable Lipschitz restriction. These are called Lipschitz trees. After an initial 1996 preprint with many properties of Lipschitz trees, including the shift operation $T^{(1)}$, the full paper by Todorčević on this topic appeared as [11. In particular, by considering embeddings between Aronszajn trees into Lipschitz ones, the paper proves that assuming $B P F A^{\aleph_{1}}$, there is no universal element in $(\mathcal{A}, \leq)$. Finally, in his book [12] Todorčević studies the class of coherent trees, which are Aronszajn trees obtained from ordinal walks, and he proves that under $M A\left(\omega_{1}\right)$ all coherent trees are Lipschitz and that such a tree $T$ embeds into $T^{(1)}$ but not the other way around. Moreover, still under $M A\left(\omega_{1}\right)$ every Aronszajn tree embeds into a coherent tree. This leads to the conclusion, Theorem 4.3.44 in [12]:

Theorem 2.1 (Todorčević) ([11]) Assuming $M A\left(\omega_{1}\right)$, there is no universal element in $(\mathcal{A}, \leq)$.

Many more results are known about $(\mathcal{A}, \leq)$, one can consult surveys [10] for earlier and [8] for more recent results.

Not that much is known about the full class $(\mathcal{T}, \leq)$. We cite the two results that we are aware of. The first one is a consistency result obtained by Mekler and Väänänen.

Theorem 2.2 ([7]) Assume $C H$ holds and $\kappa$ is a regular cardinal satisfying $\aleph_{2} \leq \kappa$ and $\kappa \leq 2^{\aleph_{1}}$. Then there is a forcing notion that preserves cofinalities (hence cardinalities) and the value of $2^{\lambda}$ for all $\lambda$, and which forces the universality number of $(\mathcal{T}, \leq)$ and the universality number of $(\mathcal{A}, \leq)$ both to be $\kappa$.

The next result, obtained by Džamonja and Väänänen, is in the presence of club guessing at $\omega_{1}$ and the failure of $C H$. It concerns weak embeddings called $\Delta$-preserving and defined by

$$
\Delta_{T_{1}}(x, y)=\Delta_{T_{2}}(f(x), f(y))
$$

Theorem 2.3 (Džamonja and Väänänen) ([3]) Suppose that

(a) there is a ladder system $\bar{C}=\left\langle c_{\delta}: \delta<\omega_{1}\right\rangle$ which guesses clubs, i.e. satisfies that for any club $E \subseteq \omega_{1}$ there are stationarily many $\delta$ such that $c_{\delta} \subseteq E$, 
(b) $\aleph_{1}<2^{\aleph_{0}}$.

Then no family of size $<2^{\aleph_{0}}$ of trees of size $\aleph_{1}$, even if we allow uncountable branches, can $\leq$-embed all members of $\mathcal{T}$ in a way that preserves $\Delta$.

Before this paper it was not known if $(\mathcal{T}, \leq)$ had a universal element under $M A\left(\omega_{1}\right)$. Our result 6.1 proves that it does not. It is not known if there is a model of set theory in which $(\mathcal{T}, \leq)$ does have a universal element. Moreover, our results (see Corollary 6.2(2)) strengthen both this conclusion and Theorem 2.1 in that they imply that under $M A\left(\omega_{1}\right)$ there is no $T$ in the larger class $(\mathcal{T}, \leq)$ which weakly embeds all elements of $(\mathcal{A}, \leq)$. It is not known if there is a model of set theory in which $(\mathcal{A}, \leq)$ or $(\mathcal{T}, \leq)$ have a universal element.

\section{Specialising triples and their basic properties}

Notation 3.1 (1) For an ordinal $\gamma<\omega_{1}$ we denote by $\mathrm{ht}(\gamma)$ the unique $\alpha$ such that $\gamma \in[\omega \alpha, \omega \alpha+\omega)$.

(2) We can without loss of generality represent $\mathcal{A}$ as the set of all normal rooted $\omega_{1}$-trees $T$ with no uncountable branches whose $\alpha$-th level is indexed by a subset of the ordinals in $[\omega \alpha, \omega \alpha+\omega)$, for $\alpha<\omega_{1}$. The root \langle\rangle is considered of level -1 .

(Recall that the requirement of being normal for a rooted tree means that if $\gamma_{0} \neq \gamma_{1}$ are of the same limit level, then there exists $\beta$ with $\beta<_{T} \gamma_{l}$ for exactly one $l<2$ ).

(3) If $T \in \mathcal{A}$ and $s, t \in T$, we denote by $s \cap_{T} t$ the maximal ordinal $\gamma$ such that $\gamma<_{T} s, t$. (Such an ordinal exists by the assumption in (2)).

If $\operatorname{ht}(x)=\alpha>\beta$, then by $x \uparrow \beta$ we denote the unique ordinal $y$ with $\operatorname{ht}(y)=\beta$ and $y<_{T} x$.

(4) For $T_{1}, T_{2} \in \mathcal{A}$ and $(x, y) \in \bigcup_{\alpha<\omega_{1}} \operatorname{lev}_{\alpha}\left(T_{1}\right) \times \operatorname{lev}_{\alpha}\left(T_{2}\right)$, we let $\alpha(x, y)$ denote the $\alpha$ such that $x \in \operatorname{lev}_{\alpha}\left(T_{1}\right)$ (and so $y \in \operatorname{lev}_{\alpha}\left(T_{2}\right)$ ).

Definition 3.2 Let $\mathcal{A}_{2}^{\mathrm{sp}}$ be the set of all triples $\left(T_{1}, T_{2}, c\right)$ where $T_{1}, T_{2} \in \mathcal{A}$ and $c$ is a function from $\bigcup_{\delta}$ limit $<\omega_{1} \operatorname{lev}_{\delta}\left(T_{1}\right) \times \operatorname{lev}_{\delta}\left(T_{2}\right)$ to $\omega$ such that

- if $c\left(x_{1}, y_{1}\right)=c\left(x_{2}, y_{2}\right)$ and $\left(x_{1}, y_{1}\right) \neq\left(x_{2}, y_{2}\right)$, then $\alpha\left(x_{1}, y_{1}\right) \neq \alpha\left(x_{2}, y_{2}\right), x_{1} \perp_{T_{1}} x_{2}$, $y_{1} \perp_{T_{2}} y_{2}$ and

$$
\Delta_{T_{1}}\left(x_{1}, x_{2}\right)>\Delta_{T_{2}}\left(y_{1}, y_{2}\right) .
$$

Remark 3.3 By the definition of $\mathcal{A}$, we have that for any $T \in \mathcal{A}$ and any $\gamma \in T, \operatorname{ht}(\gamma)$ is the same as $\mathrm{ht}_{T}(\gamma)$. The defining condition of specialising triples could have therefore been written in termes of heights, $\operatorname{ht}\left(x_{1} \cap x_{2}\right)>\operatorname{ht}\left(y_{1} \cap y_{2}\right)$.

Also note that a weak embedding is not required to be injective, but is injective on any branch of its domain. Finally, observe that every rooted Aronszajn tree is weakly bi-embeddable with a rooted normal one and hence that concentrating on rooted normal trees does not change anything from the point of view of universality results. 
The following is well known, see for example Claim 6.1 of [2].

Observation 3.4 If there exists a weak embedding from a tree $T_{1}$ to a tree $T_{2}$, then there exists one which preserves levels, namely satisfying $\mathrm{ht}_{T_{1}}(x)=\mathrm{ht}_{T_{2}}(f(x))$ for all $x \in T_{1}$.

Proof. Let $f: T_{1} \rightarrow T_{2}$ be a weak embedding. For $t \in T_{1}$, we can define $g(t)=$ $f(t)\lceil\operatorname{ht}(t)$, since $f$ being a weak embedding implies that for every such $t$ we have $\mathrm{ht}_{T_{1}}(t) \leq \mathrm{ht}_{T_{2}}(f(t))$. Now note that if $s<_{T_{1}} t$, then ht $(s)<_{T_{1}} \mathrm{ht}(t)$ and so $g(s)<_{T_{2}} g(t)$.

ㅈ․ 3

Claim 3.5 (1) If $\left(T_{1}, T_{2}, c\right) \in \mathcal{A}_{2}^{\mathrm{sp}}$ then both $T_{1}$ and $T_{2}$ are special Aronszajn trees.

(2) If $\left(T_{1}, T_{2}, c\right) \in \mathcal{A}_{2}^{\mathrm{sp}}$ then $T_{1}$ is not weakly embeddable in $T_{2}$.

(3) Every rooted normal Aronszajn tree is isomorphic to a tree in $\mathcal{A}$.

Proof. (1) Clearly, every tree in $\mathcal{A}$ is an $\omega_{1}$-tree, so $T_{1}$ and $T_{2}$ are $\omega_{1}$-trees. Let us first show that $T_{1}$ is special, so we shall define a function $d: T_{1} \rightarrow \omega$ which witnesses that.

Notice that by the assumption that $T_{2}$ is of height $\omega_{1}$, we can choose $z_{\delta}$ of height $\delta \in T_{2}$, for every limit $\delta$. Let $g: \omega \times \omega \times \omega \rightarrow \omega$ be a bijection. Every $x \in T_{1}$ is of the form $\omega \delta+\omega m+n$ for some limit ordinal $\delta$ and natural numbers $m$ and $n$. For such $x$, define $d(x)=g\left(c\left(x\left\lceil\delta, z_{\delta}\right), m, n\right)\right.$.

Suppose that $x=\omega \delta+\omega m+n, y=\omega \beta+\omega k+l$ and that $d(x)=d(y)$, while $x \neq y$. Therefore $g\left(c\left(x\left\lceil\delta, z_{\delta}\right), m, n\right)=g\left(c\left(y\left\lceil\beta, z_{\beta}\right), k, l\right)\right.\right.$ and we obtain $m=k$ and $n=l$ while $c\left(x \uparrow \delta, z_{\delta}\right)=c\left(y \uparrow \beta, z_{\beta}\right)$. Since $x \neq y$ we must have $\beta \neq \delta$ and therefore $x \uparrow \delta \neq y \uparrow \beta$. By the properties of $c$ we obtain $x \uparrow \delta \perp_{T_{1}} y \uparrow \beta$ and therefore $x \perp_{T_{1}} y$. In conclusion, $d^{-1}(\{a\})$ is an antichain, for any $a<\omega$, and therefore $d$ witnesses that $T_{1}$ is special. A similar proof shows that $T_{2}$ is special. As clearly every special $\omega_{1}$-tree is Aronszajn, the claim is proved.

(2) Suppose for a contradiction that $f$ is a weak embedding from $T_{1}$ to $T_{2}$. By Observation 3.4. we can assume that $f$ preserves levels. For each $\alpha$ limit $<\omega_{1}$ choose $x_{\alpha}$ on the $\alpha$-th level of $T_{1}$. Note that by the level preservation of $f$, the value $c\left(x_{\alpha}, f\left(x_{\alpha}\right)\right)$ is well-defined. Consider $\left\{c\left(x_{\alpha}, f\left(x_{\alpha}\right)\right): \alpha\right.$ limit $\left.<\omega_{1}\right\}$, which is necessarily a countable set since the range of $c$ is $\omega$. Hence, there must be $\alpha<\beta$ such that $c\left(x_{\alpha}, f\left(x_{\alpha}\right)\right)=c\left(x_{\beta}, f\left(x_{\beta}\right)\right)$. By the defining property of $c$ we have that $x_{\alpha} \perp_{T_{1}} x_{\beta}$.

Since $f$ is strict-order preserving we have that $f\left(x_{\alpha} \cap_{T_{1}} x_{\beta}\right)<_{T_{2}} f\left(x_{\alpha}\right), f\left(x_{\beta}\right)$ and therefore $f\left(x_{\alpha} \cap_{T_{1}} x_{\beta}\right) \leq_{T_{2}} f\left(x_{\alpha}\right) \cap_{T_{2}} f\left(x_{\beta}\right)$. However, $\operatorname{ht}\left(f\left(x_{\alpha} \cap_{T_{1}} x_{\beta}\right)\right)=\operatorname{ht}\left(x_{\alpha} \cap_{T_{1}} x_{\beta}\right)>$ $\operatorname{ht}\left(f\left(x_{\alpha}\right) \cap_{T_{2}} f\left(x_{\beta}\right)\right)$, a contradiction.

(3) Obvious.

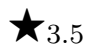

\section{Embeddings between Aronszajn trees and the non- existence of a universal element under $M A$}

This section is devoted to the proof of the following theorem. 
Theorem 4.1 For every tree $T \in \mathcal{A}$, there is a ccc forcing $\mathbb{Q}=\mathbb{Q}(T)$ and a family $\mathcal{F}=\mathcal{F}(T)$ of $\aleph_{1}$-many dense sets in $\mathbb{Q}$ such that every $\mathcal{F}$-generic filter adds a tree $T^{*}$ in $\mathcal{A}$ and a function $c$ such that $\left(T^{*}, T, c\right)$ form a specialising triple. In particular, $T^{*}$ is not weakly embeddable into $T$ and, hence, under the assumption of $M A\left(\omega_{1}\right)$ there is no Aronszajn tree universal under weak embeddings.

The latter is a result of Todorčević, see Theorem 2.1, to which our method gives an alternative proof. We shall break the proof of Theorem 4.1 into the definition of the forcing and then several lemmas needed to make the desired conclusion.

Definition 4.2 Suppose that $T \in \mathcal{A}$, we shall define a forcing notion $\mathbb{Q}=\mathbb{Q}(T)$ to consist of all $p=\left(u^{p}, v^{p},<_{p}, c^{p}\right)$ such that:

1. $u^{p} \subseteq \omega_{1} \cup\{\langle\rangle\}, v^{p} \subseteq T$ are finite and \langle\rangle$\in v^{p}$,

2. if $\alpha \in v^{p}$ then there is $\beta \in u^{p}$ with $\operatorname{ht}(\alpha)=\operatorname{ht}(\beta)$,

3. $<_{p}$ is a partial order on $u^{p}$ such that $\alpha<_{p} \beta$ implies $\mathrm{ht}(\alpha)<\mathrm{ht}(\beta)$ and which fixes $\alpha \cap_{<_{p}} \beta \in u^{p}$ for every two different elements $\alpha, \beta$ of $u^{p}$ and fixes the root \langle\rangle of $u^{p}$,

4. $c^{p}$ is a function from $\bigcup_{\delta}$ limit $<\omega_{1} \operatorname{lev}_{\delta}\left(u^{p}\right) \times \operatorname{lev}_{\delta}\left(v^{p}\right)$ to $\omega$ such that the analogue of the requirement from Definition 3.2 holds, that is:

if $c\left(x_{1}, y_{1}\right)=c\left(x_{2}, y_{2}\right)$ and $\left(x_{1}, y_{1}\right) \neq\left(x_{2}, y_{2}\right)$, then $\alpha\left(x_{1}, y_{1}\right) \neq \alpha\left(x_{2}, y_{2}\right), x_{1} \perp_{T_{1}} x_{2}$, $y_{1} \perp_{T_{2}} y_{2}$ and

$$
\operatorname{ht}\left(x_{1} \cap_{T_{1}} x_{2}\right)>\operatorname{ht}\left(y_{1} \cap_{T_{2}} y_{2}\right) .
$$

The order $p \leq q$ on $\mathbb{Q}$ is given by inclusion $u^{p} \subseteq u^{q}, v^{p} \subseteq v^{q},<_{p} \subseteq<_{q}, c^{p} \subseteq c^{q}$ with the requirement that if $p \leq q$, then the intersection and the root given by $<_{p}$ are preserved in $<_{q}$.

Lemma 4.3 There is a family $\mathcal{F}$ of $\aleph_{1}$-many dense subsets of $\mathbb{Q}$ such that for any $G$ which is $\mathcal{F}$-generic, letting

$$
T^{*}=\bigcup\left\{<_{p}: p \in G\right\} \text { and } c=\bigcup\left\{c^{p}: p \in G\right\}
$$

gives $\left(T^{*}, T, c\right) \in \mathcal{A}_{2}^{\mathrm{sp}}$.

Proof. Clearly, for any filter $G$ we have that $T^{*}$ is a partial order on $\omega_{1}$. For every $\alpha<\omega_{1}$ we have that $\operatorname{lev}_{\alpha}\left(T^{*}\right) \subseteq[\omega \alpha, \omega \alpha+\omega)$, since the same is true for every $<_{p}$ for $p \in G$. In particular, $T^{*}$ is a tree. It is a rooted tree since every $u^{p}$ for $p \in G$ has the same root. Let us observe that $T^{*}$ is normal, using the following claim.

Claim 4.4 Suppose that $\beta_{0}, \beta_{1} \in[\omega \delta, \omega \delta+\omega) \cap T^{*}$, where $\delta$ is a limit ordinal. Then there is $\alpha \in T^{*}$ such $\alpha<^{*} \beta_{l}$ for exactly one $l<2$. 
Proof. We can find $p \in G$ such that $\beta_{0}, \beta_{1} \in u^{p}$. Therefore $<_{p}$ fixes $\beta=\beta_{0} \cap_{<_{p}} \beta_{1}$ and by the definition of the order in $\mathbb{Q}$ we must have $\beta=\beta_{0} \cap_{<^{*}} \beta_{1}$. Any $\alpha<^{*} \beta_{1}$ with $\operatorname{ht}(\alpha)>\operatorname{ht}(\beta)$ satisfies the requirement.

We now show that with a judicious choice of $\mathcal{F}$ we have that $T^{*}$ is of height $\omega_{1}$.

Claim 4.5 For every $\alpha<\omega_{1}$, the set $\mathcal{D}_{\alpha}$ of all $p$ such that $u^{p}$ has an element on level $\alpha$ is dense.

Proof. Given $\alpha<\omega_{1}$, if $u^{p}$ has no elements on level $\alpha$, we shall first choose a $\gamma \in$ $[\omega \alpha, \omega \alpha+\omega)$ and extend the order $<_{p}$ to $u^{p} \cup\{\gamma\}$ by letting $\gamma$ be above the root \langle\rangle of $u^{p}$ but such that $\beta \cap_{<^{p}} \gamma=\langle\rangle$ for all $\beta \in u^{p}$. Since $u^{p}$ did not have any elements on level $\alpha$, neither does $v^{p}$, so we do not have to worry about extending $c$ to include pairs whose first coordinate is $\gamma$. 4.5

We can conclude that $T^{*}$ is a normal $\omega_{1}$-tree. The next density claim will show that $c$ is defined on all $\bigcup_{\delta}$ limit $<\omega_{1} \operatorname{lev}_{\delta}\left(T^{*}\right) \times \operatorname{lev}_{\delta}(T)$ to $\omega$ and will therefore by Claim 3.5 (1) imply that $T^{*} \in \mathcal{A}$.

Claim 4.6 Suppose that $\delta$ is a limit ordinal $<\omega_{1}$ and that there is $x$ of height $\delta$ in $u^{p}$. If $y \in T$ is of height $\delta$, then $p$ has an extension $q$ such that $y \in v^{q}$, in other words, the set $\mathcal{E}_{y}=\left\{q: y \in v^{q}\right\}$ is dense above $p$.

Proof. It suffices to let $v^{q}=v^{p} \cup\{y\}$ and to extend $c^{p}$ in a one-to-one way so that for any $x \in u^{p}$ of height $\delta$, the value of $c^{q}(x, y)$ is different from any values taken by $c^{p}$. $\star_{4.6}$

Let $\mathcal{F}$ consist of all sets $\mathcal{D}_{\alpha}$ for $\alpha<\omega_{1}$ and all sets $\mathcal{E}_{y}$ defined in and Claim 4.6 .

To finish the proof of Lemma 4.3 we have that $c$ is as required, since every $p$ satisfies the requirement from $4.2(4) . \star 4.3$

Lemma 4.7 The forcing $\mathbb{Q}(T)$ is ccc.

Proof. Suppose that $\left\langle p_{\zeta}: \zeta<\omega_{1}\right\rangle$ is a given sequence of elements of $\mathbb{Q}(T)$. By extending each $p_{\zeta}$ if necessary, we can assume that for each $\zeta$ there is an element of $v^{p_{\zeta}}$ and hence of $u^{p_{\zeta}}$ of height $\zeta$. Let $C=\left\{\zeta<\omega_{1}: \omega \zeta=\zeta\right\}$, so a club of $\omega_{1}$.

For $\zeta \in C$ let us define $q_{\zeta}=p_{\zeta}\lceil\zeta$, by which we mean:

1. $u^{q_{\zeta}}=u^{p_{\zeta}} \cap(\zeta \cup\{\langle\rangle\}), v^{q_{\zeta}}=v^{p_{\zeta}} \cap(\zeta \cup\{\langle\rangle\})$,

2. $<_{q_{\zeta}}=<_{p_{\zeta}} \uparrow u^{q_{\zeta}}$ and

3. $c^{q_{\zeta}}=c^{p_{\zeta}} \uparrow\left(u^{q_{\zeta}} \times v^{q_{\zeta}}\right)$.

There is a stationary set $S \subseteq C$, a condition $q^{*}$ and integers $n^{*}, m^{*}<\omega$ such that for every $\zeta \in S$ we have: 
1. $q_{\zeta}=q^{*}$,

2. the size of $u^{p_{\zeta}} \backslash u^{q^{*}}$ is $n^{*}$ and the size of $v^{p_{\zeta}} \backslash v^{q^{*}}$ is $m^{*}$. We enumerate them increasingly as ordinals in the form $\left\langle x_{i}^{\zeta}: i<n^{*}\right\rangle$ and $\left\langle y_{j}^{\zeta}: j<m^{*}\right\rangle$,

3. the value of $c^{p_{\zeta}}\left(x_{i}^{\zeta}, y_{j}^{\zeta}\right)$ and the fact that it is defined or not depends only on $i$ and $j$ and not on $\zeta$, and

4. letting $\gamma^{*}=\max \left(u^{q^{*}} \cup v^{q^{*}}\right)$, we have $\min \left(u^{p_{\zeta}} \backslash u^{q^{*}}\right)>\gamma^{*}+\omega$ and similarly for $v^{p_{\zeta}} \backslash v^{q^{*}}$.

By thinning further, we may assume that for every $\varepsilon<\zeta$ in $S$,

- $u^{p_{\varepsilon}} \cup v^{p_{\varepsilon}} \subseteq \zeta$,

- the unique ordinal-order-preserving functions $f_{\varepsilon, \zeta}$ from $u^{p_{\varepsilon}}$ to $u^{p_{\zeta}}$ and $g_{\varepsilon, \zeta}$ from $v^{p_{\varepsilon}}$ to $v^{p_{\zeta}}$ give rise to an isomorphism between $p_{\varepsilon}$ to $p_{\zeta}$ which fixes $q^{*}$. In particular, it maps $<_{p_{\varepsilon}}$ to $<_{p_{\zeta}}$ fixing $u^{q^{*}}$ and similarly for $<_{T}\left\lceil v^{p_{\varepsilon}}\right.$ and $<_{T} \uparrow v^{p_{\zeta}}$.

- for every $\alpha \in v^{p_{\zeta}} \backslash v^{p_{\varepsilon}}$ we have that $\alpha \uparrow_{T}\left(\gamma^{*}+\omega\right)=g_{\varepsilon, \zeta}^{-1}(\alpha) \uparrow_{T}\left(\gamma^{*}+\omega\right)$.

Let us now consider what could render two conditions $p_{\varepsilon}$ and $p_{\zeta}$ for $\varepsilon$ and $\zeta$ in $S$, incompatible. The minimum requirement on a condition $q$ with $q \geq p_{\varepsilon}, p_{\zeta}$ would be that $u^{q} \supseteq u^{p_{\varepsilon}} \cup u^{p_{\zeta}}$ and $v^{q} \supseteq v^{p_{\varepsilon}} \cup v^{p_{\zeta}}$. It may happen that there are $i<n^{*}$ and $j<m^{*}$ such that $x_{i}^{\varepsilon} \in u^{p_{\varepsilon}} \backslash \varepsilon$ and $y_{j}^{\varepsilon} \in v^{p_{\varepsilon}} \backslash \varepsilon$, so $x_{i}^{\zeta} \in u^{p_{\zeta}} \backslash \zeta$ and $y_{j}^{\zeta} \in v^{p_{\zeta}} \backslash \zeta$, such that $c\left(x_{i}^{\varepsilon}, y_{j}^{\varepsilon}\right)$ is defined, and hence $c\left(x_{i}^{\zeta}, y_{j}^{\zeta}\right)$ is defined and $c\left(x_{i}^{\zeta}, y_{j}^{\zeta}\right)=c\left(x_{i}^{\varepsilon}, y_{j}^{\varepsilon}\right)$. However, for all we know, $y_{j}^{\varepsilon}$ and $y_{j}^{\zeta}$ might be compatible in $T$ and therefore we run into a problem with the requirement (4) of Definition 4.2 of the forcing. We shall solve this difficulty by invoking the following lemma, essentially due to Baumgartner, Malitz and Reindhardt [1], here taken from Jech's book [4], where one can find a proof. In fact, although the book states the Claim in terms of Aronszajn trees, the same proof works for any tree of height and cardinality $\omega_{1}$, as long as the tree does not have an uncountable branch. We shall use that fact in $\$ 5$, so we state the claim in these terms.

Claim 4.8 ([4, Lemma 16.18) If $\mathbf{T}$ is tree of height and cardinality $\omega_{1}$ with no uncountable branches and $W$ is an uncountable collection of finite pairwise disjoint subsets of $\mathbf{T}$, then there exist $s, s^{\prime} \in W$ such that any $x \in s$ is incomparable with any $y \in s^{\prime}$.

We can now apply Claim 4.8 to find $\varepsilon<\zeta$ both in $S$ such that any $y_{j}^{\varepsilon}$ is incomparable with any $y_{j^{\prime}}^{\zeta}$. Now we claim that $p_{\varepsilon}$ and $p_{\zeta}$ are compatible. Let us start by defining $v=v^{p_{\varepsilon}} \cup v^{p_{\zeta}}$ and $u^{\prime}=u^{p_{\varepsilon}} \cup u^{p_{\zeta}}$. In order to get a condition we shall have to extend $u^{\prime}$ and also define $<$, but note already that if $\alpha \in v$, then there is an element of height ht $(\alpha)$ in $u^{\prime}$, since the analogue is true about $u^{p_{\varepsilon}}$ and $u^{p_{\zeta}}$. So conditions 1 . and 2. of Definition 4.2 are easy to fulfil and it is condition 4. that is difficult. Once we fulfil it, that Condition 3. will follow from the proof. 
Our choices so far imply that $c=c^{p_{\varepsilon}} \cup c^{p_{\zeta}}$ is a well defined function. In order to use it to fulfil condition 4. of Definition 4.2, we have to check through all the pairs $\left(x_{1}, y_{1}\right) \neq\left(x_{2}, y_{2}\right)$ in $\bigcup_{\delta} \operatorname{limit}<\omega_{1} \operatorname{lev}_{\delta}\left(u^{\prime}\right) \times \operatorname{lev}_{\delta}(v)$ such that $c\left(x_{1}, y_{1}\right)=c\left(x_{2}, y_{2}\right)$. If $\left(x_{1}, y_{1}\right),\left(x_{2}, y_{2}\right)$ are both in $\operatorname{dom}\left(c^{p_{\varepsilon}}\right)$ or both are in $\operatorname{dom}\left(c^{p_{\zeta}}\right)$, then the condition 4 . is satisfied for them, so the interesting case is when they are not.

Therefore $\alpha\left(x_{1}, y_{1}\right) \neq \alpha\left(x_{2}, y_{2}\right)$, and let us suppose, without loss of generality, that $\alpha\left(x_{1}, y_{1}\right)<\alpha\left(x_{2}, y_{2}\right)$. Then necessarily $\left(x_{1}, y_{1}\right) \in \operatorname{dom}\left(c^{p_{\varepsilon}}\right) \backslash \operatorname{dom}\left(c^{p_{\zeta}}\right)$ and $\left(x_{2}, y_{2}\right) \in$ $\operatorname{dom}\left(c^{p_{\zeta}}\right) \backslash \operatorname{dom}\left(c^{p_{\varepsilon}}\right)$. We have assured that this implies that $y_{1}$ and $y_{2}$ are incompatible in $T$. Let $\gamma=\operatorname{ht}\left(y_{1} \cap_{T} y_{2}\right)$, so $\gamma<\alpha\left(x_{1}, y_{1}\right)$. So far we know nothing about $x_{1} \cap x_{2}$ since neither $<_{p_{\varepsilon}}$ nor $<_{p_{\zeta}}$ have the pair $\left(x_{1}, x_{2}\right)$ in its domain. Knowing that $\alpha\left(x_{1}, y_{1}\right)$ is a limit ordinal, we are going to choose a successor ordinal $\beta_{x_{1}, x_{2}}$ above $\max \left(\gamma, \gamma^{*}\right)$ and below $\alpha\left(x_{1}, y_{1}\right)$ and an ordinal $w_{x_{1}, x_{2}}$ of height $\beta_{x_{1}, x_{2}}$ which is not $<_{p_{\varepsilon}}$ above any element of $u^{p^{\varepsilon}}$. We shall add $w_{x_{1}, x_{2}}$ to $u^{\prime}$ and declare $w_{x_{1}, x_{2}}=x_{1} \cap_{<} x_{2}$. We do this for all pairs relevant to condition 4 ., by induction on the number of such pairs, each time avoiding all interaction with what we have already chosen. At the end let $u$ be the union of $u^{\prime}$ and the set of all such $w_{x_{1}, x_{2}}$. Since the new elements are all of successor height, this will not bring us in danger of creating new instances of condition 4. Finally, to fulfil condition 3. we need to extend $<_{p_{\varepsilon}} \cup<_{p_{\zeta}}$ to a partial order $<$ on $u$ which will respect the commitments on $\bigcap_{<}$which we have just made, which is possible by the way we chose $\beta_{x_{1}, x_{2}}$.

Then the condition $q=(u, v,<, c)$ is a common extension of $p_{\varepsilon}, p_{\zeta} . \star 4.7$

Proof. (of Theorem 4.1) To finish the proof, we suppose that we are in a model of $M A\left(\omega_{1}\right)$ and that $T$ is an Aronszajn tree. Without generality, passing to a weakly biembeddable copy and adding a root if necessary, we can assume that $T$ is rooted and normal.Then by forcing by the ccc forcing $\mathbb{Q}(T)$ (Lemma 4.7) and intersecting $\aleph_{1}$ many dense sets $\mathcal{D}_{\alpha}$ for $\alpha<\omega_{1}$ (Claim 4.5) and $\mathcal{E}_{y}$ for $y \in T$ (Claim 4.6), we obtain that the generic Aronszajn tree $T^{*}$ does not weakly embed into $T$ (Lemma 4.3 and Claim 3.5(2)). Therefore, $T$ is not universal, and since $T$ is arbitrary, the theorem is proved.

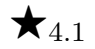

Remark 4.9 Theorem 4.1 gives another proof of the main result of [1], which is that under $M A\left(\omega_{1}\right)$ all Aronszajn trees are special and another proof of Theorem 2.1.

\section{$5 \quad$ Embedding wide Aronszajn trees into Aronszajn trees}

This section is devoted to the proof of the following theorem:

Theorem 5.1 For every tree $T \in \mathcal{T}$, there is a ccc forcing $\mathbb{P}=\mathbb{P}(T)$ and a family $\mathcal{H}=\mathcal{H}(T)$ of $\aleph_{1}$ many dense sets in $\mathbb{P}$, such that every $\mathcal{H}$-generic filter adds a tree in $\mathcal{A}$ into which $T$ weakly embeds. In particular, under the assumption of $M A\left(\omega_{1}\right)$, the class $\mathcal{A}$ is cofinal in the class $(\mathcal{T}, \leq)$. 
Following the pattern from Section \$4, we shall break the proof into the definition of the forcing and then several lemmas needed to make the desired conclusion. The forcing is dual to the one in $\$ 4$, in the sense that we now start with a tree $T$ in $\mathcal{T}$ and generically add an Aronszajn tree that $T$ weakly embeds to. We use the control function $c$ to make sure that the generic tree does not have an uncountable branch.

For the definition of the forcing, we represent every $T \in \mathcal{T}$ by an isomorphic copy which is a subtree of ${ }^{\omega_{1}>} \omega_{1}$.

Definition 5.2 Suppose that $T \subseteq \omega_{1}>\omega_{1}$ is a tree of size $\aleph_{1}$ and with no uncountable branches, we define a forcing notion $\mathbb{P}=\mathbb{P}(T)$ to consist of all $p=\left(u^{p}, v^{p},<_{p}, f^{p}, c^{p}\right)$ such that:

1. $u^{p} \subseteq T, v^{p} \subseteq \omega_{1}$ are finite and \langle\rangle$\in u^{p}$,

2. $u^{p}$ is closed under intersections,

3. $<_{p}$ is a partial order on $v^{p}$,

4. $f^{p}$ is a surjective weak embedding from $\left(u^{p}, \subset\right)$ onto $\left(v^{p},<_{p}\right)$,

5. for every $\eta \in u^{p}$, we have $\mathrm{ht}\left(f^{p}(\eta)\right)=\lg (\eta)$ (notice that $\lg (\eta)=\mathrm{ht}_{T}(\eta)$, since $\eta$ is a sequence of ordinals),

6. $c^{p}$ is a function from $v^{p}$ into $w$ such that

$$
\alpha<_{p} \beta \Longrightarrow c^{p}(\alpha) \neq c^{p}(\beta)
$$

The order $p \leq q$ on $\mathbb{P}$ is given by inclusion: $u^{p} \subseteq u^{q}, v^{p} \subseteq v^{q},<_{p} \subseteq<_{q}$ and $c^{p} \subseteq c^{q}$.

Lemma 5.3 There is a family $\mathcal{H}$ of $\aleph_{1}$-many dense subsets of $\mathbb{P}$ such that for any $G$ which is $\mathcal{H}$-generic, letting

$$
T^{*}=\bigcup\left\{<_{p}: p \in G\right\}, f=\bigcup\left\{f^{p}: p \in G\right\}, \text { and } c=\bigcup\left\{c^{p}: p \in G\right\},
$$

we have that $T^{*}$ is an Aronszajn tree, $f$ is a level-preserving weak embedding of $T$ into $T^{*}, c: T^{*} \rightarrow \omega$ and $\alpha<_{T^{*}} \beta \Longrightarrow c(\alpha) \neq c(\beta)$.

Proof. Clearly, for any filter $G$ the set $T^{*}$ is a partial order on a subset of $\omega_{1}, c$ is a well defined function into $\omega$ and $f$ is a function from a subset of $T$ into $T^{*}$ which is a weak embedding of its domain into its range. In addition, $f$ is level-preserving in the sense that for all $\eta \in \operatorname{dom}(f)$ we have ht $(f(\eta))=\lg (\eta)$ and $c$ satisfies $\alpha<_{T^{*}} \beta \Longrightarrow c(\alpha) \neq c(\beta)$. To finish the proof of the Lemma, we prove the following three claims.

Claim 5.4 (Density Claim) There is a set $\mathcal{H}$ of $\aleph_{1}$ many dense subsets of $\mathbb{P}$ such that if $G$ is $\mathcal{H}$-generic, then domain of $f^{*}$ is $T$. 
Proof. Let $\rho \in T$, we shall show that $\mathcal{E}_{\rho}=\left\{p \in \mathbb{P}: \rho \in \operatorname{dom}\left(f^{p}\right)\right\}$ is dense. Suppose that $p \in P$ is given and suppose that $p \notin \mathcal{E}_{\rho}$. We shall define an extension $q$ of $p$ which is in $\mathcal{E}_{\rho}$. Let us define $u_{0}^{q}=u^{p} \cup\{\rho\}$. Let $\alpha=\lg (\rho)$. We shall first extend $f^{p}$ to $u_{0}^{q}$. For the ease of reading, we divide the proof into steps.

(1) The first case is that either there is no $\tau \in u^{p}$ with $\rho \subset \tau$, or that there are such $\tau$ but there is no $\tau^{\prime}, \rho^{\prime} \in u^{p}$ such that $\lg \left(\rho^{\prime}\right)=\alpha, \rho^{\prime} \subset \tau^{\prime}$ and $f^{p}\left(\tau^{\prime}\right)=f^{p}(\tau)$. In this case choose $\gamma \in[\omega \alpha, \omega \alpha+\omega) \backslash v^{p}$ and define $v_{0}^{q}=v^{p} \cup\{\gamma\}, f^{q}(\rho)=\gamma$. Let $\gamma>_{q} \beta$ for any $\beta=f^{p}(\sigma)$ for some $\sigma \subset \rho$ and $\gamma<_{q} \delta$ for any $\delta=f^{p}(\tau)$ for $\rho \subset \tau$ and $\tau \in u^{p}$. Then the relation $<_{q}$ is a partial order. We let $c^{q}(\gamma)$ be any value in $\omega$ not taken by $c^{p}$.

(2) This step is the main point. It is that there is $\tau \in u^{p}$ with $\rho \subset \tau$ and $\tau^{\prime}, \rho^{\prime} \in u^{p}$ such that $\lg \left(\rho^{\prime}\right)=\alpha, \rho^{\prime} \subset \tau^{\prime}$ and $f^{p}\left(\tau^{\prime}\right)=f^{p}(\tau)$. In this case we shall have $v_{0}^{q}=v^{p},<_{q}^{0}=<_{p}$ and $c^{q}=c^{p}$, so let us show how to extend $f^{p}$ to $f^{q}$. Let $\tau$ be of the least length among all $\tau \mathrm{s}$ as in the assumption of this case. We are then obliged to let $f^{q}(\rho)=f^{p}\left(\rho^{\prime}\right)$, since $f^{p}(\tau)$ can have only one restriction to the level $\alpha$ and $f^{p}\left(\rho^{\prime}\right)$ is already such a restriction. Note that for any $\tau^{\prime \prime}, \rho^{\prime \prime} \in u^{p}$ such that $\lg \left(\rho^{\prime \prime}\right)=\alpha, \rho^{\prime \prime} \subset \tau^{\prime \prime}, f^{p}\left(\tau^{\prime \prime}\right)=f^{p}(\tau)$, we must have $f^{p}\left(\rho^{\prime \prime}\right)=f^{p}\left(\rho^{\prime}\right)$ since $f^{p}$ is a weak embedding. However, there is a possible problem: there could be $\sigma, \sigma^{\prime}$ and $\rho^{\prime \prime}$ such that $\lg \left(\rho^{\prime \prime}\right)=\alpha, \rho \subset \sigma, \rho^{\prime \prime} \subset \sigma^{\prime}, f^{p}(\sigma)=f^{p}\left(\sigma^{\prime}\right)$, which would force us to have $f^{p}(\rho)=f^{p}\left(\rho^{\prime \prime}\right)$, but maybe $f^{p}\left(\rho^{\prime \prime}\right) \neq f^{p}\left(\rho^{\prime}\right)$. Luckily, this cannot happen since $u^{p}$ is closed under intersections, so for any such $\sigma$ we would have $\rho=\sigma \cap \tau \in u^{p}$, which is not the case. In fact, any $\sigma \in u^{p}$ with $\rho \subset \sigma$ must satisfy $\tau \subseteq \sigma$.

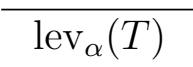

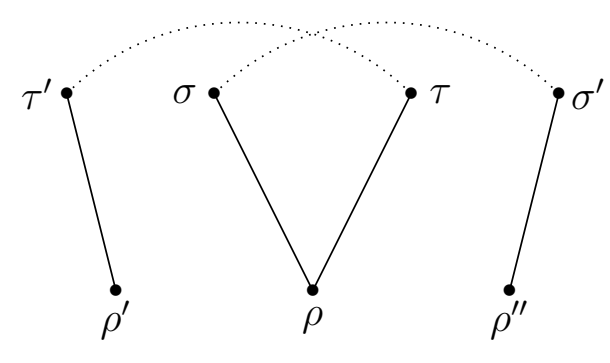

Main point

(3) Now we know what $f^{q}(\rho)$ is and we have to discuss the closure under intersections. If there is $\tau \in u^{p}$ with $\rho \subset \tau$, then taking such $\tau$ of minimal length, we have that for every $\sigma \in u^{p}, \rho \cap \sigma=\tau \cap \sigma$, by the minimality of the length of $\tau$ and the fact that $u^{p}$ is closed under intersections. In this case we let $u^{q}=u_{0}^{q}$ and $v^{q}=v_{0}^{q}$ and we are done. So suppose that there is no such $\tau$. Let $\sigma \in u^{p}$ be the longest initial segment of $\rho$ which is in $u^{p}$, which exists since $u^{p}$ is finite and it contains \langle\rangle . Then, if there are intersections of the elements of $u_{0}^{q}$ which are not already be in $u_{0}^{q}$, they must be of the form $\tau \cap \rho$ for some $\tau \in u^{p}$ with $\sigma \subset \tau$. Moreover, by the closure of $u^{p}$ under intersections and the choice of $\sigma$, there is a single $\tau \in u^{p}$ of least length which satisfies $\tau \subseteq \tau^{\prime}$ for any other such $\tau^{\prime}$. We then add $\tau \cap \rho$ to $u_{0}^{q}$ to form $u^{q}$ and we note that this set is now closed under intersections. If $u^{q}=u_{0}^{q}$, then we are done. Otherwise, $u^{q} \backslash u_{0}^{q}$ is a singleton and 
let $\beta$ be such that the unique element of $u^{q} \backslash u_{0}^{q}$ of length $\beta$. We then choose an ordinal $\gamma_{\beta} \in[\omega, \omega \beta+\omega) \backslash \operatorname{ran}\left(f^{p}\right)$ and we let $f^{p}(\sigma)<_{q} \gamma_{\beta}<_{q} f^{q}(\rho)$. We extend $<_{q}$ by transitivity. Finally we choose an element $c_{\beta} \in \omega \backslash \operatorname{ran}\left(c_{0}^{q}\right)$ and let $c^{q}\left(\gamma_{\beta}\right)=c_{\beta}$.

To finish the proof of the claim, let $\mathcal{H}$ consist of all $\mathcal{E}_{\rho}$ for $\rho \in T$. $\star_{5.4}$

Claim 5.5 For every $\alpha<\omega_{1}$ we have that $\operatorname{lev}_{\alpha}\left(T^{*}\right) \subseteq[\omega \alpha, \omega \alpha+\omega)$ and $T^{*}$ has size $\aleph_{1}$.

Proof. It follows from the definition of the forcing that $\operatorname{ran}\left(f^{p}\left\lceil\left(\operatorname{lev}_{\alpha}(T)\right) \subseteq[\omega \alpha, \omega \alpha+\omega)\right.\right.$ for every $p \in \mathbb{P}$. That every $\operatorname{lev}_{\alpha}(T)$ is non-empty follows from Claim 5.4 .5.5

We can conclude that $T^{*}$ is an $\omega_{1}$-tree. By genericity we have that the domain of $c$ is $T^{*}$ and that $c: T^{*} \rightarrow \omega$ satisfies $\alpha<_{p} \beta \Longrightarrow c^{p}(\alpha) \neq c^{p}(\beta)$.

Claim 5.6 $T^{*}$ has no uncountable branch.

Proof. This is an easy consequence of the properties of $c$, namely $c$ is 1-1 on any branch, and its range is a subset of $\omega . \star_{5.6}$

Therefore $T^{*}$ is an Aronszajn tree. To finish the proof of the lemma, it remains to verify that $f: T \rightarrow T^{*}$ is a weak embedding, which follows from the genericity. $\star_{5.3}$

Lemma 5.7 The forcing $\mathbb{P}(T)$ is ccc.

Proof. Recalling that the elements $\rho$ of $T$ are functions from a countable ordinal to $\omega_{1}$, we shall use the notation $\rho\lceil\alpha$ to denote the restriction of $\rho$ to $\lg (\rho) \cap \alpha=\max \{\lg (\rho), \alpha\}$ and $\rho(\beta)$ for the value of $\rho$ at $\beta \in \lg (\rho)$. Also observe that $\lg (\rho)=\operatorname{dom}(\rho)$.

Suppose that $\left\langle p_{\zeta}: \zeta<\omega_{1}\right\rangle$ is a given sequence of elements of $\mathbb{P}(T)$. By extending each $p_{\zeta}$ if necessary, using the density of the sets $\mathcal{E}_{\rho}$ from Claim 5.4, we can assume that for each $\zeta<\omega_{1}$ :

(a) there is an element of $u^{p_{\zeta}}$ and hence of $v^{p_{\zeta}}$ of height $\zeta$, and that

(b) for every $\rho \in u^{p_{\zeta}}$ and every $\beta<\lg (\rho)$ such that there is an element of $u^{p_{\zeta}}$ of height $\beta$, the point $\rho \uparrow \beta$ is in $u^{p_{\zeta}}$.

Let

$$
C=\left\{\zeta<\omega_{1}: \omega \zeta=\zeta \text { and }(\forall \varepsilon<\zeta) \max \left\{\lg (\rho), \rho(\alpha): \rho \in u^{p_{\varepsilon}}, \alpha<\lg (\rho)\right\}<\zeta\right\},
$$

so $C$ is a club of $\omega_{1}$ consisting of limit ordinals. By extending again if necessary, we shall require that for every $\zeta \in C$, there is an element in $u^{p_{\zeta}}$ of height in $(0, \zeta)$. For $\zeta \in C$ let us define $q_{\zeta}=p_{\zeta}\lceil\zeta$, by which we mean:

1. $u^{q_{\zeta}}=u^{p_{\zeta}} \cap<\zeta \omega_{1}, v^{q_{\zeta}}=v^{p_{\zeta}} \cap \zeta$, 
2. $<_{q_{\zeta}}=<_{p_{\zeta}}\left\lceil v^{q_{\zeta}}\right.$ and

3. $f^{q^{\zeta}}=f^{p^{\zeta}}\left\lceil u^{q_{\zeta}}, c^{q_{\zeta}}=c^{p_{\zeta}}\left\lceil v^{q_{\zeta}}\right.\right.$.

Applying the Fodor Lemma and the Delta-System Lemma, we obtain a stationary set $S \subseteq C$ such that:

1. for every $\zeta \in S$ we have: $v^{q_{\zeta}}=v^{*},<_{q_{\zeta}}=<^{*}, c^{q_{\zeta}}=c^{*}$ are fixed,

2. the sets $u^{q_{\zeta}}$ form a $\Delta$-system with root $u^{*}$,

3. for every $\varepsilon<\zeta \in S$ there is a level-preserving order isomorphism $\varphi_{\varepsilon, \zeta}$ from $u^{q_{\varepsilon}}$ to $u^{q_{\zeta}}$ which is identity on $u^{*}:^{2}$

4. for every $\varepsilon<\zeta \in S, f^{q_{\varepsilon}}=f^{q_{\zeta}} \circ \varphi_{\varepsilon, \zeta}$,

5. for every $\varepsilon<\zeta \in S$, there is an order preserving isomorphism $\psi_{\varepsilon, \zeta}$ from $\left(u^{p_{\varepsilon}}, \subseteq\right)$ to $\left(u^{p_{\zeta}}, \subseteq\right)$ which extends $\varphi_{\varepsilon, \zeta}$ and such that $f^{p_{\epsilon}}=f^{p_{\zeta}} \circ \psi_{\varepsilon, \zeta}$,

6. for every $\varepsilon<\zeta \in S$, there is an order preserving isomorphism $i_{\varepsilon, \zeta}$ from $\left(v^{p_{\varepsilon}},<_{p_{\varepsilon}}\right)$ to $\left(v^{p_{\zeta}},<_{q_{\zeta}}\right)$ which is identity on $v^{*}$.

By the fact that there is an element of height $\zeta$ in $u^{p_{\zeta}}$, we have that each $u^{p_{\zeta}} \backslash u^{q_{\zeta}} \neq \emptyset$. Since \langle\rangle$\in u^{q_{\zeta}}$ we have that $u^{q_{\zeta}} \neq \emptyset$ for all $\zeta$, but even more so, $u^{q_{\zeta}}$ has an element of height in $(0, \zeta)$. Let $\alpha_{1}=\max \left\{\lg (\rho): \rho \in u^{q_{\zeta}}\right\}$ and $\alpha_{0}=\min \left\{\lg (\rho): \rho \neq\langle\rangle \in u^{q_{\zeta}}\right\}$. Since $\zeta$ is an element of $C$, it is a limit ordinal. The set $u^{q_{\zeta}}$ is finite set and for every $\rho \in u^{q_{\zeta}}$ the length $\lg (r h o)<\zeta$, so we have that $\alpha_{1}<\zeta$. By the choice of $\varphi_{\varepsilon, \zeta}$, the choice of $\alpha_{0}$ and $\alpha_{1}$ does not depend on $\zeta$. Finally let $\delta=\min (C) \backslash \alpha_{1}$.

Our requirements and the fact that $T$ does not have an uncountable branch imply that we can use Claim 4.8 to find $\varepsilon<\zeta \in S \backslash \delta$ such that for every $\rho \in u^{p_{\varepsilon}} \backslash u^{*}$ and $\sigma \in u^{p_{\zeta}} \backslash u^{*}, \rho$ and $\sigma$ are incomparable. We shall find a common extension of $p_{\varepsilon}$ and $p_{\zeta}$.

We first define $u_{0}=u^{p_{\varepsilon}} \cup u^{p_{\zeta}}$. We also define $f_{0}=f^{p_{\varepsilon}} \cup f^{p_{\zeta}}$, which is well defined by the assumptions of the $\Delta$-system, and, similarly, $c_{0}=c^{p_{\varepsilon}} \cup c^{p_{\zeta}}$. We also simply let $<_{0}=<_{p_{\varepsilon}} \cup<_{p_{\zeta}}$, which still gives a partial order by the choice of $\varepsilon$ and $\zeta$. Specifically, $<_{0}$ makes any element of $v^{p_{\varepsilon}}$ incomparable to any element of and $v^{p_{\zeta}}$, which conforms to the fact that any element of $u^{p_{\varepsilon}} \backslash u^{*}$ is incomparable to any element of $u^{p_{\zeta}} \backslash u^{*}$.

The only problem is that $u_{0}$ is not necessarily closed under intersections. Let us analyse what type of intersection can occur and what we need to add to make $u_{0}$ closed under intersections.

Let $\rho, \tau \in u_{0}$. If $\rho, \tau \in u^{p_{\varepsilon}}$ or $\rho, \tau \in u^{p_{\zeta}}$ then $\rho \cap \tau \in u^{*}$. Let us now suppose that we are dealing with some $\rho \in u^{p_{\varepsilon}} \backslash u^{p_{\zeta}}$ and $\tau \in u^{p_{\zeta}} \backslash u^{p_{\varepsilon}}$, the other case is symmetric.

Case 1. $\lg (\rho \cap \tau)<\alpha_{0}$. We handle all instances of such $\rho$ and $\tau$ simultaneously.

Using that $\rho \nmid \alpha_{0} \in u^{p_{\varepsilon}}$ and $\tau\left\lceil\alpha_{0} \in u^{p_{\zeta}}\right.$, it suffices to consider the case $\lg (\rho)=$ $\lg (\tau)=\alpha_{0}$.

\footnotetext{
${ }^{2}$ Since $u^{p_{\varepsilon}}$ and $u^{p_{\zeta}}$ are closed under intersections, $\varphi_{\varepsilon, \zeta}$ necessarily preserves intersections.
} 
Let $\sigma_{0}, \ldots, \sigma_{n}$ be all $\sigma=\rho \cap \tau$ obtained in this way. We choose for each $i<n+1$ distinct $f\left(\sigma_{i}\right)$ with $\operatorname{ht}\left(f\left(\sigma_{i}\right)\right)=\lg \left(\sigma_{i}\right)$ (note that necessarily $f\left(\sigma_{i}\right) \in \omega_{1} \backslash \operatorname{ran}\left(f_{0}\right)$ ) and distinct $c_{i}$ in $\omega \backslash \operatorname{ran}\left(c_{0}\right)$. Extend $u_{0}$ by adding all $\sigma_{0}, \ldots, \sigma_{n}$ and $v_{0}$ by adding all $f\left(\sigma_{i}\right)$. Extend $<_{0}$ to a transitive order on $v_{0}$ which satisfies $f\left(\sigma_{i}\right)<_{0} f(\eta)$ when $\sigma_{i} \subset \eta$ for some $\rho \in u^{p_{\varepsilon}} \cup u^{p_{\zeta}}$. This is possible because there are no elements of $u_{0}$ of length $<\alpha_{0}$. Extend $c_{0}$ to include the values $c_{i}=c\left(f\left(\sigma_{i}\right)\right)$ as above. Call the resulting tuple $\left(u_{1}, v_{1},<_{1}, f_{1}, c_{1}\right)$. Case 2. $\lg (\rho \cap \tau) \in\left[\alpha_{0}, \alpha_{1}\right)$. We handle all instances of such $\rho$ and $\tau$ simultaneously.

Let $\sigma=\rho \cap \tau$. By our assumption (b) we can assume that $\rho \in u^{q_{\varepsilon}} \backslash u^{*}$ and $\tau \in u^{q_{\zeta}} \backslash u^{*}$ are of the least possible length with the intersection $\sigma$. By the fact that $\varphi_{\varepsilon, \zeta}$ preserves both order and height, another application of (b) lets us assume that ht $(\rho)=\operatorname{ht}(\tau)$. The possible dangerous configuration is that there are $\rho^{\prime} \in u^{q_{\varepsilon}} \backslash u^{*}, \tau^{\prime} \in u^{q_{\zeta}} \backslash u^{*}$ of length $\lg (\rho)$ and $\sigma^{\prime} \in u^{q_{\varepsilon}} \backslash u^{*}, \sigma^{\prime \prime} \in u^{q_{\zeta}} \backslash u^{*}$ of length $\lg (\sigma)$ such that $\sigma^{\prime} \subset \rho^{\prime}$ and $\sigma^{\prime \prime} \subset \tau^{\prime}$, $f^{p_{\varepsilon}}\left(\rho^{\prime}\right)=f^{p_{\varepsilon}}(\rho)=f^{p_{\zeta}}(\tau)=f^{p_{\zeta}}\left(\tau^{\prime}\right)$, yet $f^{p_{\varepsilon}}\left(\sigma^{\prime}\right) \neq f^{p_{\zeta}}\left(\sigma^{\prime \prime}\right)$.

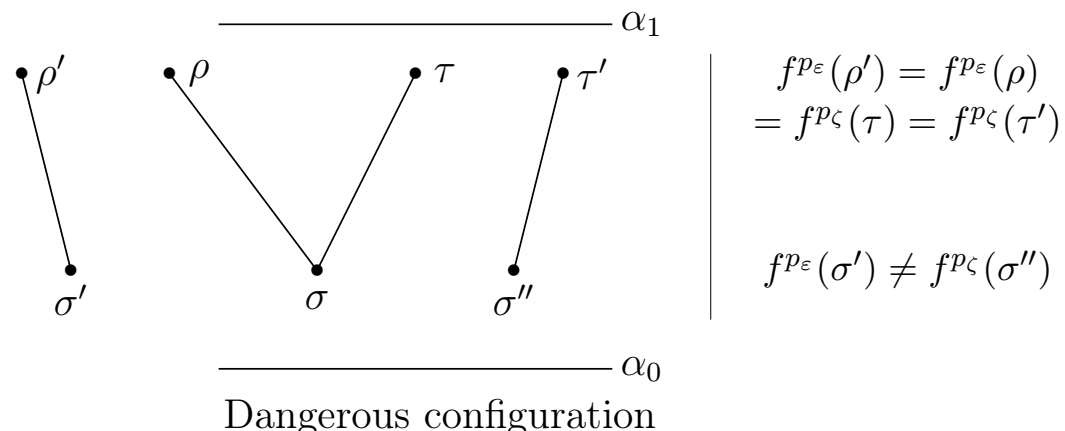

If there were such points we would not be able to extend $f_{1}$ to $\sigma$ and keep it a weak embedding. Luckily, this cannot happen since if there were to be any elements $\eta$ of $u^{q_{\varepsilon}}$ of length $\lg (\sigma)$, then by the fact that $u^{p_{\varepsilon}}$ satisfies the assumption (b), $\sigma=\rho \uparrow \lg (\eta)$ would already be $u^{p_{\varepsilon}}$, so in $u^{q_{\varepsilon}}$.

This analysis shows that we can proceed as in Case 1 to extend $\left(u_{1}, v_{1},<_{1}, f_{1}, c_{1}\right)$ to $\left(u_{2}, v_{2},<_{2}, f_{2}, c_{2}\right)$ which is closed under all intersections of Case 2 and satisfies other requirements of being a condition. Note that $\left(u_{2}, v_{2},<_{2}, f_{2}, c_{2}\right)$ remains closed under the intersections of length $<\alpha_{0}$.

Case 3. $\lg (\rho \cap \tau)=\alpha_{1}$.

Let $\sigma=\rho \cap \tau$. We have that $\sigma=\rho \uparrow \alpha_{1} \in u^{p_{\varepsilon}}$ and $\sigma=\tau \uparrow \alpha_{1} \in u^{p_{\zeta}}$ and hence $\sigma \in u^{*}$, a contradiction.

Case 4. $\lg (\rho \cap \tau)>\alpha_{1}$.

Let $\sigma=\rho \cap \tau$. By the choice of $S$, we have that $u^{p_{\zeta}}$ does not have any elements of length $\lg (\sigma)$ and by the fact that $u^{p_{\varepsilon}}$ is closed under restrictions, since $\sigma=\rho \uparrow \lg (\sigma)$, we have that there are no elements of $u^{p_{\varepsilon}}$ of length $\lg (\sigma)$ either. Hence we can proceed like in Case 1. Once we are done closing under intersections of this type, we finally obtain a common extension of $p_{\varepsilon}, p_{\zeta} \cdot \star 5.7$

Proof. (of Theorem 5.1) The proof follows by putting the lemmas together. $\star 5.1$ 


\section{Conclusion}

Putting the results of Section $\$ 4$ and Section $\$ 5$ together, we obtain our main theorem, as follows.

Theorem 6.1 Under $M A\left(\omega_{1}\right)$, there is no wide Aronszajn tree universal under weak embeddings.

Proof. Assume $M A\left(\omega_{1}\right)$ and suppose for a contradiction that $T$ is a universal element in $(\mathcal{T}, \leq)$. By Theorem 5.1, there is an Aronszajn tree $T^{\prime}$ such that $T \leq T^{\prime}$, so $T^{\prime}$ is universal in $(\mathcal{T}, \leq)$ and so in $(\mathcal{A}, \leq)$. However, by Theorem $4.1(\mathcal{A}, \leq)$ does not have a universal element, a contradiction. $\star 6.1$

We also remark that putting our results together with the results of Todorčević mentioned in \$4.9, gives the first part of the following Corollary 6.2. The second part of the corollary improves Todorčević 's theorem 2.1 and our Main Theorem 6.1.

Corollary 6.2 Assume $M A\left(\omega_{1}\right)$. Then:

(1) The class $\mathcal{L}$ of Lipschitz trees and the class of coherent trees are cofinal in the class $(\mathcal{T}, \leq)$

(2) There is no element of $(\mathcal{T}, \leq)$ which suffices to weakly embed all Aronszajn trees

Proof. (1) Our Main Theorem 6.1 shows that under $M A\left(\omega_{1}\right)$, the class or Aronszajn trees is cofinal in the class of wide Aronszajn trees. On the other hand, Todorčević in $\S 4$ of [12] has shown that under the same assumptions, the class of coherent trees is cofinal in the class of all Aronszajn trees and that every coherent tree is Lipschitz.

(2) This is a direct consequence of Theorem 5.1 . $\star 6.2$

\section{References}

[1] James E. Baumgartner, Jerome Malitz, and William Reinhardt. Embedding trees in the rationals. Proc. Natl. Acad. Sci. USA, 67(4):1748-1753, 1970.

[2] Mirna Džamonja and Jouko Väänänen. Chain models, trees of singular cardinality and dynamic EF-games. J. Math. Log., 11(1):61-85, 2011.

[3] Mirna Džamonja and Jouko Väänänen. A family of trees with no uncountable branches. Topology Proc., 28(1):113-132, 2004. Spring Topology and Dynamical Systems Conference.

[4] Thomas Jech. Set Theory. Springer-Verlag, Berlin Heidelberg, 3rd millenium edition, 2003. 
[5] Đuro (Georges) Kurepa. Transformations monotones des ensembles partiallement ordonnes. Compt. Rend. Acad. Sci, 205:1033-1035, 1937.

[6] Đuro (Georges) Kurepa. Transformations monotones des ensembles partiallement ordonnes. In Aleksandar Ivić, Zlatko Mamuzić, Žarko (editor-in-chief) Mijalović, and Stevo Todorčević, editors, Selected papers of Duro Kurepa, pages 165-186. Matematički Institut u Beogradu, 1996.

[7] Alan Mekler and Jouko Väänänen. Trees and $\Pi_{1}^{1}$-subsets of ${ }^{\omega_{1}} \omega_{1}$. J. Symb. Log., 58(3):1052-1070, 1993.

[8] Justin Tatch Moore. Structural analysis of Aronszajn trees. In Costas Dimitracopoulos, Ludomir Newelski, Dag Normann, and John Steel, editors, Proceedings of the 2005 Logic Colloquium in Athens, Greece, volume 28 of Lecture Notes in Logic, pages 85-107, 2006.

[9] Roman Sikorski. Remarks on some topological spaces of high power. Fundamenta Mathematicae, 37:125-136, 1949.

[10] Stevo Todorčević. Trees and linearly ordered sets. In Kenneth Kunen and Jerry E. Vaughan, editors, Handbook of set-theoretic topology, pages 235-293. North Holland, Amsterdam, 1984.

[11] Stevo Todorčević. Lipschitz maps on trees. Journal of the Institute of Mathematics of Jussieu, 6(3):527-556, 2007.

[12] Stevo Todorčević. Walks on ordinals and their characteristics, volume 263 of Progress in Mathematics. Birkhäuser Verlag, Basel, 2007. 